\section{Arquitectura como tecnología de construcción de la realidad social}

XURXo M. AYÁn VILA

Laboratorio de Arqueoloxía, Instituto de Estudios Galegos Padre Sarmiento (CSIC-XuGa) / UA LaFC (IIT, USC)

\begin{abstract}
Resumen
Se desarrolla textualmente el enfoque arqueotectónico reflejado en el póster homónimo presentado en el / Seminario Internacional de Arqueología de la Arquitectura con el que se pretendía mostrar gráficamente la potencialidad y aplicabilidad al registro arqueológico de determinadas analíticas y metodologías desarrolladas en el ámbito interdisciplinar de la Arqueología de la Arquitectura. Partiendo de una propuesta teórica concreta definida a partir de la Arqueología del Paisaje postprocesual, abordamos el análisis sintáctico del espacio doméstico a nivel de poblado como herramienta básica para alcanzar una definición de modelos o patrones de organización espacial que fundamenten una interpretación sociológica de las formaciones socioculturales del pasado.

En este sentido mostramos un ejemplo práctico concreto: la aplicación de analíticas espaciales a un poblado fortificado galaicorromano prácticamente excavado en extensión permitirá esbozar el modelo de espacialidad vigente en ese período ahondando en la problemática de la estabilidad y pervivencia de tradiciones arquitectónicas de la Edad del Hierro conjuntamente con el factor de cambio y ruptura impuestos por la romanización del NW peninsular.
\end{abstract}

Palabras Clave: Arqueología de la Arquitectura; Arqueología del Paisaje; Arqueología del espacio doméstico; castros; sociedad galaicorromana.

\begin{abstract}
This text develops the archaeotectonic approach reflected in the poster of the same name at the $1^{\text {st }}$ International Archaeology of Architecture Seminar, with a view to showing graphically the potential and applicability to archaeology of certain analyses and methodologies developed in the interdisciplinary field of Archaeology of Architecture. Working from a specific theoretical proposal defined from post-procesual landscape archaeology, we approach the syntactic analysis of domestic space at town level as a basic instrument in defining models or patterns of spatial organization which form the basis of a sociological interpretation of socio-cultural formations from the past.

We provide a concrete practical example of this: the application of spatial analyses to an extensively excavated Galician-Roman fortified town will allow us to sketch a model of spatiality present in this period, exploring the problems of stability and survival of architectonic traditions from the Iron Age along with the factor of change and rupture imposed by the Romanization of the northwest of the Iberian peninsular.
\end{abstract}

Key words: Archaeology of Architecture; Landscape Archaeology; Domestic space archaeology; hill-forts / Iron-Age settlements; Galician-Roman society.

\section{LA ARQUEOLOGÍA DEL ESPACIO CONSTRUIDO}

La integración de este póster en el citado Seminario se explica como una consecuencia lógica del enfoque asumido por nuestro grupo de trabajo en lo que se refiere a la relación Arqueología y Arquitectura. Desde la Arqueología del Paisaje desarrollamos un marco teórico que bajo la denominación genérica de Arqueotectura intenta definir una Arqueología del Espacio Construido de carácter integral que aboga por una perspectiva diacrónica en el tratamiento de la información arquitectónica, aplicable tanto a la Prehistoria Reciente, como a la Arquitectura protohistórica e histórica, configurando una perspectiva que supere la aproximación clásica que identifica la Arqueología de la Arquitectura con la metodología concreta de la lectura estratigráfica de paramentos (MAŃANA et alii, 2002; AYÁn et alii, 2003).

Nuestra propuesta no pretende invalidar otros modos de investigar el registro arquitectónico, simplemente se ha tratado de trazar los aspectos básicos de una línea de investigación multidisciplinar, con el fin de obtener de los espacios construidos la mayor información posible, una forma de analizar el registro que trata de buscar regularidades y cuyo medio de contrastación de las hipótesis debe bastante a la Arqueología interpretativa estructuralista (CRIADO BOADO, 1993, 1999; JOHNSON, 1993; HODDER, 1994).

Desde nuestro punto de vista la arquitectura es una tecnología y herramienta básica para la reproducción social, un catalizador y a la vez producto de la acción social, una herramienta cultural que construye el paisaje social. La arquitectura reproduce el patrón de racionalidad de una sociedad, generando una estructura espacial, unas relaciones espaciales que reflejan una determinada lógica social. En consecuencia un edificio no se reduce a un mero objeto arquitectónico, es una entidad material que desempeña un rol activo en la constitución social de la realidad arqueológica.

\section{ARQUEOLOGÍA DE LA ARQUITECTURA Y ESPACIO DOMÉSTICO}

El análisis del espacio doméstico a escala microespacial y su concepción como objeto de estudio de la investigación prehistórica fue una consecuencia directa del desarrollo de los planteamientos procesuales en el ámbito anglosajón. Para los nuevos arqueólogos las unidades arquitectónicas ubicadas dentro de los asentamientos (nivel micro) daban la clave para comprender el patrón de subsistencia y la estructura social de las comunidades que habían construido esas edificaciones. Es por ello que los programas de investigación privilegiasen la identificación de áreas de actividad en el seno de los asentamientos, con el objeto de definir la funcionalidad de los diferentes espacios y aproximar una 
interpretación global de los poblados. El análisis de la distribución y asociaciones de artefactos dentro de las estructuras arquitectónicas, las actividades y función de los espacios eran la base para una interpretación sociológica del espacio arquitectónico (CLARKE, 1972; UCKO et alii, 1972).

Este enfoque motivó el desarrollo activo de dos tendencias, la Settlement Archaeology y la Archaeology of Household Activities (WILK y RAHTJE, 1982; Allison, 1999) que promocionaron una notable innovación metodológica, diseñándose nuevas técnicas de análisis que permitieron la definición de modelos espaciales. El surgimiento posterior de los diferentes enfoques postprocesuales incrementó el marco analítico para acercarse al estudio de los espacios domésticos configurándose un notable bagaje metodológico (SÁNCHEZ, 1998) dentro del que destacan el análisis funcional y simbólico (BLANTON, 1994; HODDER, 1994), el análisis formal (CHING, 1995; BAKER, 1998; CRIADO, 1999) y el análisis sintáctico del espacio (HILlier y HaNSON, 1984).

La aproximación al espacio doméstico que proponemos aplicar al registro arqueológico del NW pretende aplicar un modelo analítico que combina la descripción formal de las estructuras con la aplicación de las herramientas metodológicas citadas ${ }^{1}$ con el objetivo de maximizar el enorme potencial de los restos arquitectónicos.

\section{EL ANÁLISIS ARQUEOTECTÓNICO}

El espacio construido se presenta como el producto o efecto de la acción social. Por lo tanto constituye un paisaje ${ }^{2}$ cultural en sentido amplio; así por ejemplo, la forma arquitectónica aparece interrelacionada con variables sociológicas como la familia, el estilo de vida, la solidaridad intergrupal o el sistema de poder. De este modo, se puede definir como un producto humano que utiliza una realidad dada (el espacio físico) para crear una realidad nueva: el espacio habitacional y, por consiguiente, social, a la que se confiere un significado simbólico.

\footnotetext{
${ }^{1}$ Un desarrollo descriptivo de estas metodologías se recoge en el artículo de nuestra compañera Patricia Mañana por lo que no nos extenderemos en este aspecto concreto.

2 Seguimos la definición de paisaje propuesta por Criado (1999: 5): producto socio-cultural creado por la objetivación, sobre el medio y en términos espaciales, de la acción social tanto de carácter material como imaginario.

3 Se entiende por análisis formal el análisis de las formas materiales concretas que constituyen el paisaje, tanto las naturales (fisiográficas) como las artificiales (elementos de Cultura Material, monumentos...), sin introducir un sentido extraño a ellos. Es por lo tanto un tipo de práctica deconstructiva que, cuando tiene éxito, describe el objeto de estudio desde dentro de si mismo (CRIADO, 1999: 20).
}

Dicho producto se compone de diferentes entidades formales, que se proyectan espacialmente, son visibles, por lo que pueden ser percibidas y descritas por la observación arqueológica. El análisis de las relaciones espaciales significativas entre las entidades del registro, permite reconstruir mínimamente su contexto y, en menor medida, su sentido originales. El estudio de estas relaciones espaciales entre elementos, esto es, la estructura espacial, permite acercarnos no sólo a la lógica espacial de una determinada comunidad sino también a la propia lógica social del espacio (HILLIER y HANSON, 1984).

Dicha estructura espacial es generada por una sociedad concreta por medio de unas determinadas tecnologías espaciales y arquitectónicas que reproducen el patrón de racionalidad imperante y obedecen a un determinado sistema de representaciones. Éste aparece reflejado en todos los ámbitos de la acción social, los cuales están determinados por códigos espaciales compatibles y semejantes entre sí. Por lo tanto los diferentes productos materiales de una formación social (arquitectura, cerámica, etc...) presentan relaciones de compatibilidad, configurando una regularidad espacial, ya que obedecen a la misma estrategia de construcción del espacio social.

La problemática fundamental que presenta el estudio arqueotectónico del espacio doméstico radica en el procedimiento a utilizar para emprender la contrastación empírica de la propuesta teórica anteriormente enunciada. En este sentido, en el estudio específico de la arquitectura doméstica castreña (AYÁn, 2001) utilizamos de manera conjunta las siguientes metodologías analíticas.

\section{Análisis constructivo}

Se parte de un estudio descriptivo de las construcciones para poner de manifiesto las características genéricas de la arquitectura doméstica del yacimiento objeto de estudio. Este análisis tipológico y constructivo permitirá aislar y definir las formas que definen esa arquitectura.

\section{Análisis formal}

Este análisis formal ${ }^{3}$, centrado para nuestro caso en un nivel espacial concreto del paisaje castreño como es la arquitectura de las unidades domésticas, se centra en la configuración espacial concreta del registro arquitectónico, de su patrón de emplazamiento en el espacio circundante, articulación interna, función social, condiciones de visibilidad y condiciones de visibilización, patrón de movimiento y accesibilidad. El análisis formal de todas y cada una de estas dimensiones nos permitirá establecer el patrón formal y seguidamente un modelo hipotético de la organización espacial propia de la arquitectura doméstica castreña. 
Estas formas arquitectónicas modelan el espacio interior en que se vive, el espacio doméstico; por lo tanto generan una serie de relaciones espaciales y pautas de organización interna que responden a las exigencias funcionales impuestas por los habitantes/constructores del yacimiento. Mediante el análisis formal podremos llegar a vislumbrar los principios ordenadores-jerarquizadores del espacio arquitectónico e interpretar la hipotética funcionalidad de las estructuras que lo delimitan.

\section{Análisis sintáctico del espacio}

Una vez definidas las estructuras arquitectónicas y reseñadas las relaciones espaciales a que dan lugar, se puede llevar a cabo una aproximación al significado social subyacente. La aplicación de las siguientes metodologías ayudan a deconstruir la lógica social que preside ese ordenamiento espacial:

-El análisis gamma cuantifica las profundidades y permeabilidades de los espacios, así como la facilidad de acceso, valorando el grado de dependencia existente entre ellos. Por lo tanto, permiten valorar la relación existente entre el espacio exterior a la vivienda, ésta y el interior habitacional de la misma. Mediante el diagrama de permeabilidad se pueden ver las relaciones sintácticas entre los espacios, representados cada uno de ellos por medio de un círculo, y colocando en la misma horizontal los espacios que tienen igual valor (FOSTER, 1989).

-El análisis de la circulación - materializado igualmente en un diagrama - identifica el orden perceptivo de una construcción a través del movimiento de sus espacios, reconociendo espacios preeminentes en el esquema general de la configuración del recorrido.

-El análisis de la visibilidad permite definir el grado de privacidad de los espacios de una construcción arquitectónica cerrada. Se realiza en función de la situación del individuo que percibe el interior de la estructura desde un punto de vista concreto, que se corresponde con el centro del umbral de acceso a cada estancia. En este sentido los diferentes grados de visibilidad desde el exterior son definidos por la ubicación y tamaño del vano de la puerta de entrada, así como por los propios paramentos murarios. Para conocer la ubicación hipotética de cada uno de ellos, se trazan dos líneas imaginarias desde el centro de cada umbral, y se dirigen hacia los límites establecidos por las barreras arquitectónicas; los espacios que no se ven supuestamente serían los de carácter más restringido al observador, hasta que le fuese permitido el paso al interior de la estructura.

Mediante analíticas de este estilo se puede llegar a identificar patrones de espacialidad que se pueden interpretar correlacionándolos con la estructura socioeconómica y el aparato simbólico manejado por las comunidades que erigieron, usaron y transformaron las construcciones arquitectónicas estudiadas.

\section{UN EJEMPLO DE ANÁLISIS ARQUEOTECTÓNICO. EL CASTRO DE VILADONGA (CASTRO DE REI, LUGO)}

\section{Contexto historiográfico}

Para el ámbito gallego apenas contamos con análisis microespaciales ${ }^{4}$ aplicados a castros, de ahí que la investigación sobre el espacio doméstico y la vida cotidiana en la Edad del Hierro esté aún por desarrollar. Esta carencia explica la concepción predominante en la actualidad sobre el hábitat castreño como así se ha señalado en anteriores trabajos (FERnÁNDEZ PosSE, 1998; AYÁn, 2002). De acuerdo con este enfoque clásico (ROMERO Masiá, 1976; ACUÑa CASTROVIEJO, 1996; CARBALlO, 1996) las comunidades castreñas presentarían una escasa jerarquización y complejización social, como así parece manifestarse en la enorme dispersión del poblamiento en el territorio, la aparente homogeneidad del registro arquitectónico o en la nula articulación interna de los poblados. Sólo la llegada de Roma al NW conllevaría la urbanización de unos poblados caóticos carentes de lógica espacial ${ }^{5}$.

Desde nuestra óptica creemos que esta visión se encuentra directamente condicionada por una práctica arqueológica que ha repetido sus limitaciones durante décadas. Es necesario establecer las bases de una relectura de un registro, eso sí, sesgado ${ }^{6}$, pero que mantiene un potencial informativo capaz de ampliar el conocimiento arqueológico. A ello queremos contribuir con el análisis arqueotectónico propuesto. No obstante, somos conscientes de las enormes limitaciones con que se puede encontrar, para el ámbito castreño, una estrategia de investigación como la aquí esbozada.

El análisis arqueotectónico propuesto sólo puede ser aplicado en su integridad a yacimientos con un amplio

\footnotetext{
${ }^{4}$ Ejemplos de este tipo de analíticas son los trabajos de A. de la Peña en Santa Trega (DE LA PENAA, 1998), los estudios en yacimientos de la zona de Las Médulas llevados a cabo por el equipo de Sánchez Palencia (FerNÁNdez Posse y SÁnChez Palencia, 1998) o nuestro propio trabajo en el castro de Elviña (AYÁn, 2001).

5 Para una crítica de esta perspectiva véase el amplio trabajo de AlmagroGorbea (1994).

${ }^{6}$ Entre las limitaciones del registro arqueológico castreño cabe destacar la inexistencia de una secuencia o periodización convencionalmente aceptada, la visión tipológica y formalista de las construcciones que apenas ha incidido en aspectos como la funcionalidad de las estancias, así como el análisis separado de construcciones y ajuares que dificulta la contextualización de los espacios domésticos.
} 

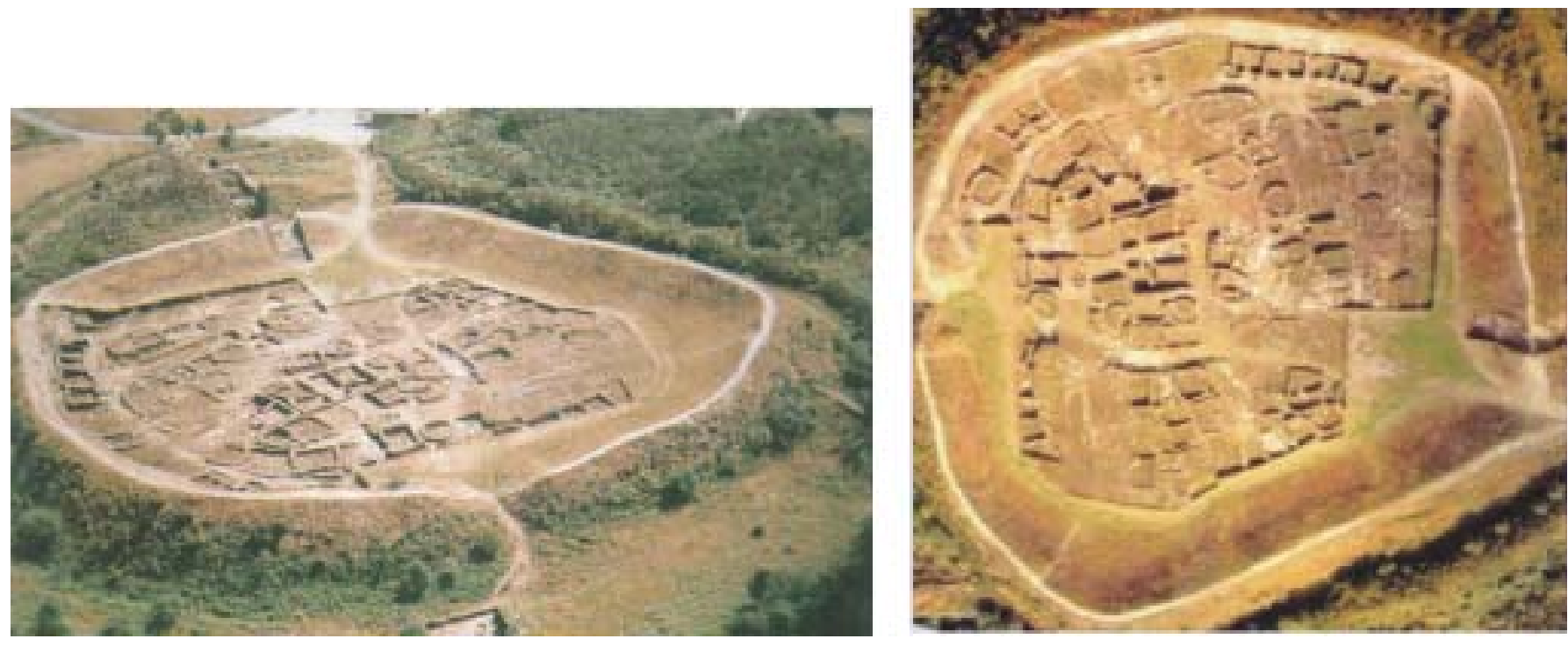

Fig. 1. Fotografía aérea del castro de Viladonga (ARIAS y DURÁN, 1996)

sector excavado de su espacio habitacional. Este es el caso del castro de Viladonga, yacimiento significativo para comprender el paso del patrón de espacialidad prerromano a un nueva modelación del espacio doméstico en época galaicorromana.

\section{El yacimiento}

El castro de Viladonga ${ }^{7}$ (Castro de Rei, Lugo), ubicado en la corona de un monte que domina la zona NE de la Terra Chá, presenta una acrópolis de forma circular y defendida por muralla pétrea, fosos y parapetos, con una extensión próxima a los $10.000 \mathrm{~m}^{2}$. El yacimiento fue objeto de 16 campañas de excavación desde 1971 (dirigidas por Chamoso Lamas hasta 1978 y posteriormente por F. Arias Vilas) dejando al descubierto un conjunto arquitectónico monumental utilizado como espacio habitacional de manera continuada y estable en época tardorromana, entre finales del siglo II y finales del siglo $\mathrm{V}$ d. C. si bien existen datos de una ocupación anterior (CHAMOSO LAMAS, 1977; ARIAS, 1985; ARIAS, 2000; ARIAS y DURÁN, 1996).

El yacimiento cuenta con un museo de sitio dependiente de la Xunta de Galicia y dirigido por F. Arias Vilas. Desde esta institución se ha desarrollado recientemente un trabajo de revisión del registro arqueológico generado por las intervenciones antiguas centrándose entre otros aspectos en el análisis pormenorizado de la organización espacial del

\footnotetext{
7 http://www.aaviladonga.es/

8 En gran medida seguimos el modelo de análisis sintáctico desarrollado por Hillier y Hanson. Véase el trabajo de Chapman como aplicación práctica de esta metodología en otro contexto prehistórico (CHAPMAN, 1990).
}

poblado (LLANA y VARELA, 1999, 2000, 2001). Algunas de las hipótesis esbozadas por estos autores se corroboran mediante la aplicación de un análisis arqueotectónico.

\section{Análisis sintáctico del espacio doméstico}

En las figuras que acompañan el presente texto recogemos gráficamente las analíticas ${ }^{8}$ llevadas a cabo a partir de la planimetría disponible sobre el yacimiento (ARIAS, 1985). El mapa de articulación axial del poblado nos muestra claramente la existencia de dos ejes N-S y E-W (a modo de cardo y decumano). Estas dos líneas - que completan el marco espacial definido por la propia muralla a la que se ciñen otras unidades arquitectónicas - no sólo articulan el ordenamiento interno al definir el recorrido circulatorio básico, sino que también definen una serie de conjuntos arquitectónicos significativos o barrios. Existe una organización espacial interna basada no sólo en la adaptación a las condiciones del terreno sino también en un planteamiento urbanístico de naturaleza ortogonal, con vías de circulación en función de las cuales se ordenan las unidades habitacionales.

A su vez el mapa de articulación convexa define los espacios públicos-semipúblicos del asentamiento y el grado de permeabilidad directa de esas unidades con respecto a éstos. El análisis gamma y el análisis de visibilidad de las unidades habitacionales completa a escala micro la visión general sobre el asentamiento en una triple vertiente: a) refleja las restricciones impuestas al libre recorrido circulatorio; b) muestra la segmentación interna y la jerarquización de los diferentes espacios o estancias, y c) permite elaborar el mapa general de accesos del yacimiento.

Las construcciones domésticas delimitan un espacio arquitectónico propio en el que se dan una serie de relacio- 
nes espaciales asimétricas. Se constata una organización espacial distribuida (sólo se puede acceder de una manera) que manifiesta un control estricto del acceso: para llegar a $B$ desde el exterior es necesario pasar antes por $A$, que actúa como un filtro de acceso y una barrera a la libre circulación por el interior de la vivienda. Los atrios pavimentados, los machones salientes, la presencia de umbrales perfectamente trabajados y los vestíbulos constituyen herramientas arquitectónicas que actúan como manifestación material de una estrategia de impermeabilización del espacio habitacional. Asimismo se registran espacios distribuidores de la circulación, propios de estructuras con una notable compartimentación interna, constatándose un acentuado control del recorrido posible dentro de las estructuras habitacionales. De este modo, se advierte la restricción establecida por la unidad social para preservar el espacio habitacional.

Las viviendas del castro son un conjunto arquitectónico individualizado y cerrado, conformado por tres espacios fundamentales que se pueden definir como espacio público, semipúblico/semiprivado y privado. A este respecto el análisis de visibilidad muestra una articulación perceptiva de los controles de acceso que definen en la práctica estos tres espacios.

En consecuencia se obtiene un patrón espacial que en gran medida se explica por la existencia de un modelo social de articulación del espacio doméstico interpretable dentro del contexto de la sociedad galaicorromana.

\section{Viladonga. Un ejemplo del modelo de espacialidad galaicorromano}

Los resultados del análisis corroboran el modelo espacial esbozado a partir de la aplicación de esta misma metodología al castro de Elviña (A Coruña) (AYán, 2001). Este yacimiento, datable en el cambio de era, experimentó - como otros castros costeros reconvertidos en lugares centralesuna honda transformación con el impacto romanizador, con unos cambios en la organización interna que muestran una sociedad inmersa en un proceso de aculturación que culmina con el asentamiento en las zonas llanas y el abandono de los poblados fortificados en el siglo II d. C.

Dentro de este contexto el castro de Viladonga es un ejemplo de pervivencia en época bajoimperial del modelo de poblado fortificado (con la crisis del s. III d. C. tuvo lugar en la Gallaecia un proceso de reocupación de antiguos castros, si bien el algunas zonas del interior se dio una continuidad de hábitat) pudiéndose considerar como el paradigma del modelo de espacialidad imperante en los asentamientos galaicorromanos.

Este patrón espacial consolida una serie de variaciones significativas que reforman la uniforme tradición arquitec- tónica doméstica de la Edad del Hierro del NW. En primer lugar la utilización de la planta cuadrangular supone una maximización del espacio interior de la vivienda; no olvidemos que la construcción circular constituye una solución arquitectónica poco rentable en lo que se refiere al aprovechamiento de la superficie habitacional. Asimismo la línea recta permite una distribución más ordenada de las edificaciones, haciendo posible la práctica del adosamiento de estructuras mediante el empleo de paredes medianeras. Todo ello revierte en una mayor racionalización del espacio construido en la línea del modelo urbanístico mediterráneo.

La forma rectangular favorece igualmente la aparición de divisiones internas dentro de las construcciones domésticas. La compartimentación del espacio interno implica la delimitación de diferentes áreas, la presencia de límites espaciales físicos y, en consecuencia, un mayor grado de privacidad, preservado por un control acusado del acceso a esas estancias. La segmentanción del espacio doméstico indica una complejización socioespacial notable con respecto al espacio único de la vivienda circular.

A su vez, no se da un acceso directo desde el exterior al interior del espacio doméstico; aparecen ahora estructuras arquitectónicas que mediatizan y limitan la libre circulación, actuando como mecanismos de control del espacio de la entrada. Otro aspecto novedoso a tener en cuenta es la ruptura de la disposición radial de las áreas de actividad en torno a un punto marcado por el hogar central. La lareira comienza a ubicarse de manera sistemática junto a la pared, ya sea en un lateral al lado de la puerta o enfrente de ella.

Se incrementan las labores de aterrazamiento y preparación del terreno y se favorece la circulación en el interior del recinto con rebajes, escaleras y pequeños viales pavimentados. Asimismo, se registran edificios de carácter público, diferenciados claramente del resto de edificaciones.

Se mejoran notablemente las técnicas constructivas (generalización del uso de instrumental de hierro en las labores de cantería, desarrollo de la carpintería). El aparejo de mampostería de los muros es objeto de mayor cuidado, utilizándose en algunos casos argamasa de mortero en las juntas; la cara interna de las paredes recibe también un mejor tratamiento, extendiéndose la práctica decorativa del enlucido. Asimismo se generaliza la introducción de nuevas soluciones arquitectónicas, como es el empleo de la teja para la cubierta de las construcciones rectangulares (el tejado va haciéndose un hueco al lado de la tradicional cubierta de colmo).

Estos cambios en la arquitectura van acompañados a su vez de novedades en el ajuar doméstico de las viviendas: la introducción del molino circular giratorio, la generaliza- 


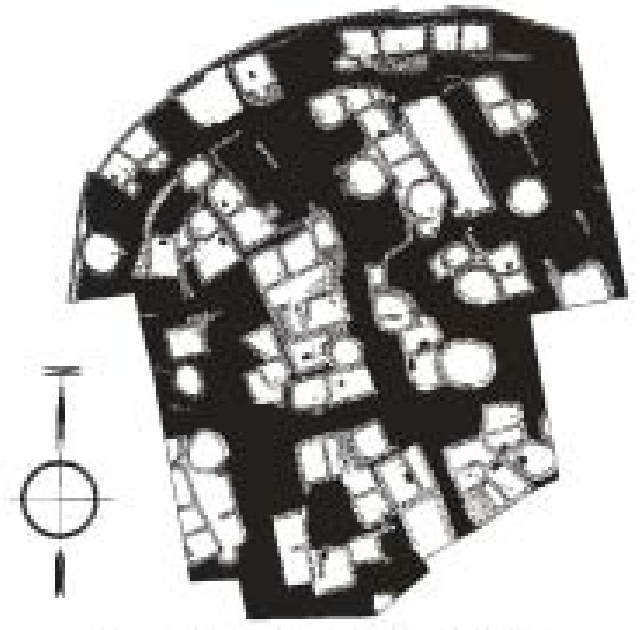

Espacio abierto dentro del poblado (y) exchoye el espacis de las cictulas primarias (pivade)

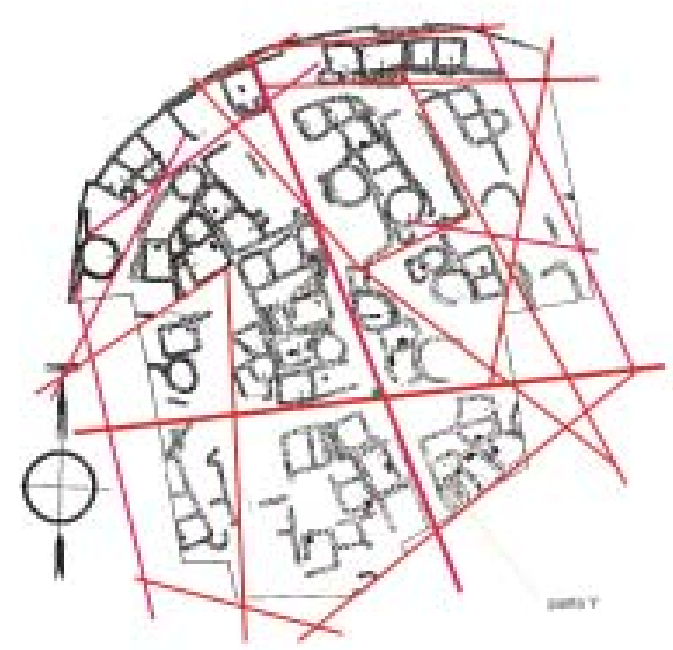

Mapa axial del poblado muestra la organizacion respecto al enterior forgarización global del sistema)

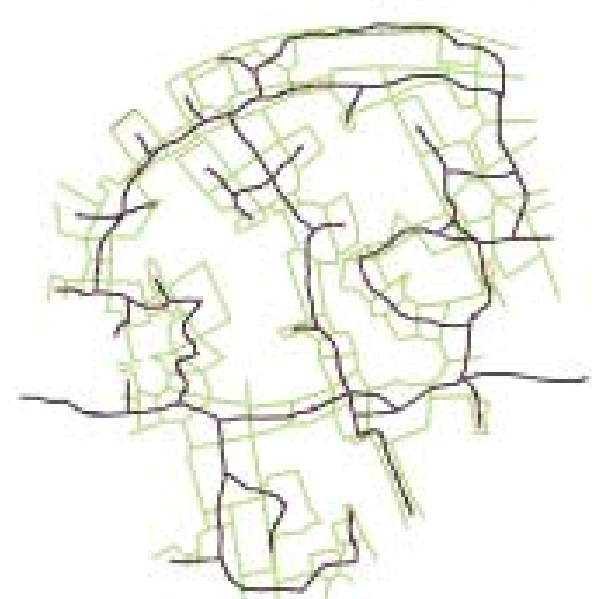

Recoerido circulatoria dentro del poblado hace relerendia a la relscion de provimidad ortre los

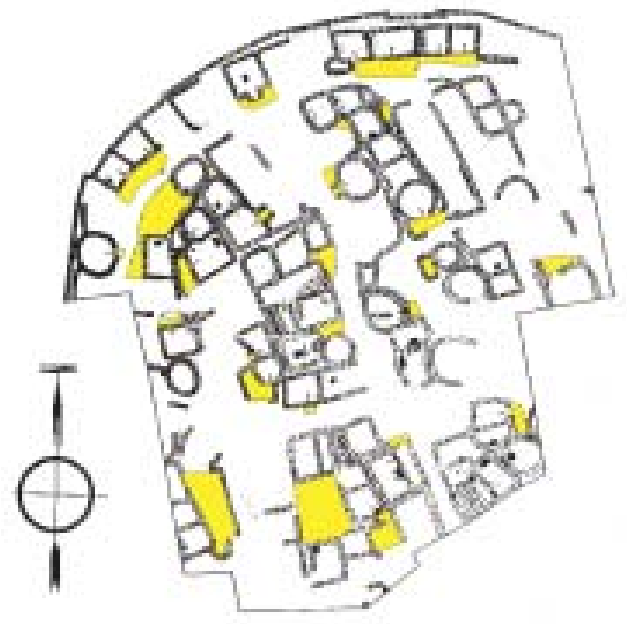

Espacia semipublioo dentro del poblado resata el espacio fronierizo ente el espacio pobico y el pritrado

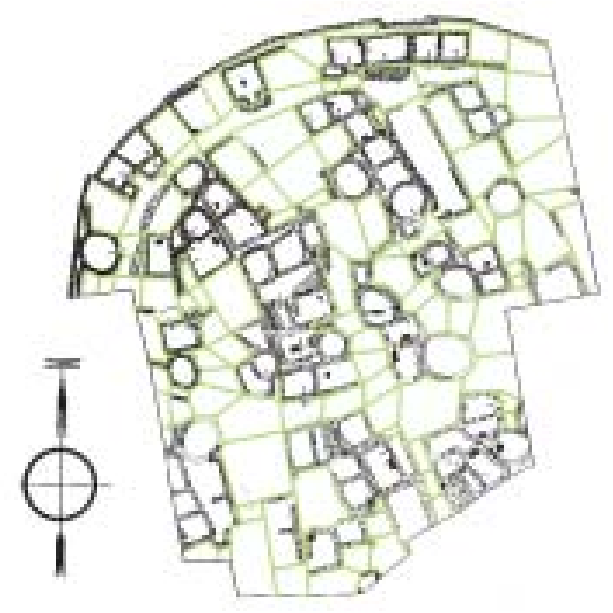

Mapa cocrere del potlado muestra la orgarizacibn reepecto las oldulas primanioss (organizacion local sistema)

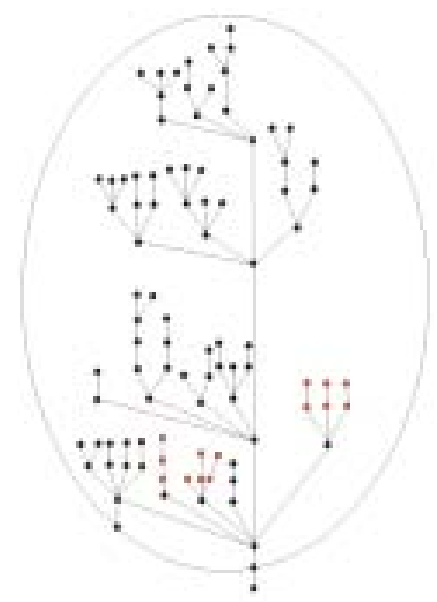

Diagrama del accesos a las cedulas primarios del poblado esquematiza la permeablidod de las vaiendas

Fig. 2. Análisis arqueotectónico del espacio doméstico del castro 

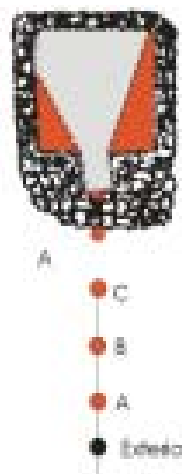

Estuchara 1
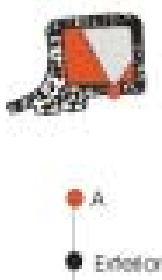

Eatruchua 4
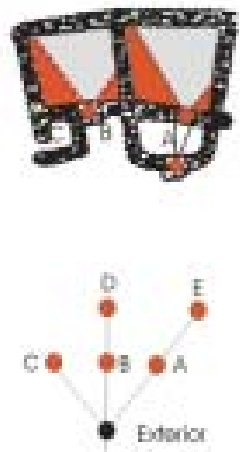

Estructura 2
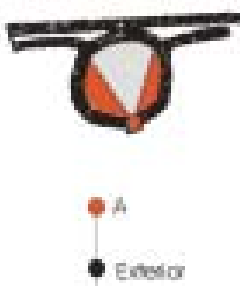

Estructura
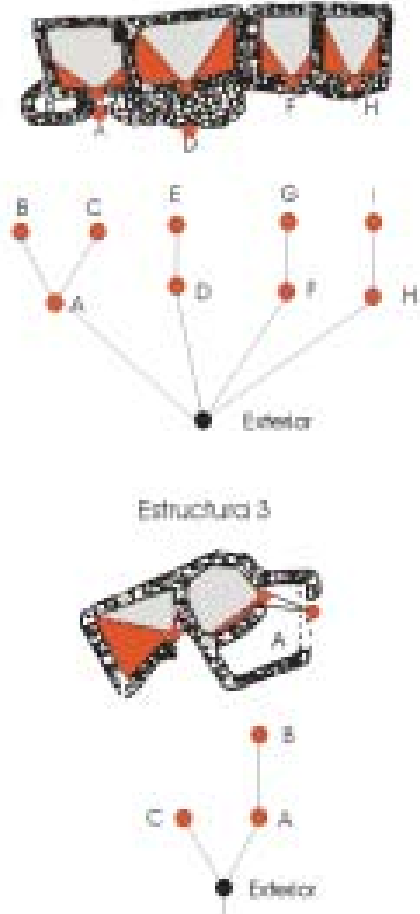

Estuctura 6

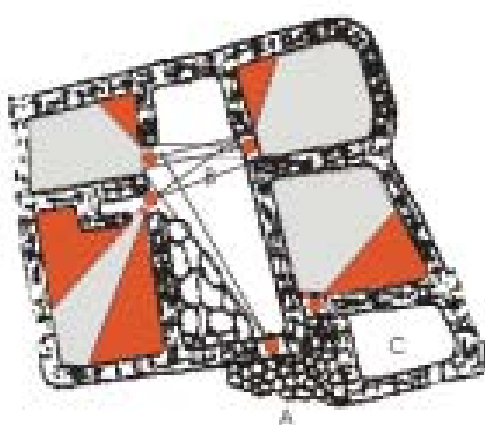

Estuctura $?$

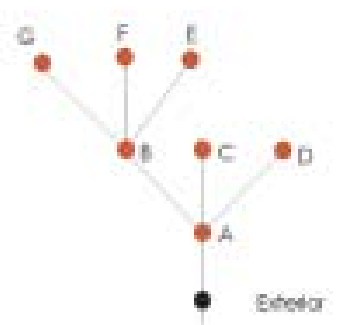

Fig. 3. Análisis gamma y análisis de visibilidad aplicados a estructuras domésticas del castro

ción de la cerámica de mesa romana (común y terra sigillata) o la presencia de ánforas de almacenamiento en el interior de las casas, son todos ellos indicios claros de nuevos hábitos domésticos cotidianos.

Estas discontinuidades señalan la incipiente configuración de un nuevo modelo de espacialidad en los castros que siguen siendo utilizados como lugar de habitación durante el proceso de conformación de la sociedad galaicorromana. Son, por lo tanto, claros signos de aculturación de una sociedad indígena sobre la que actúa la política socioeconómica promovida por Roma.

En este sentido se percibe un proceso ambivalente de continuidad y ruptura; por un lado, la arquitectura de Viladonga refleja la transformación producida en el patrón de racionalidad y en la estructura social de estas comunidades; por otro lado, muestra también la persistencia de la fuerte tradición arquitectónica indígena. La interpretación de este fenómeno debe abordarse por lo tanto en una doble dirección. Cabe plantearse la posibilidad de que en este período se mantuviese todavía a grandes rasgos el modelo conceptual del espacio doméstico prerromano. Los cambios afectan de hecho a la arquitectura doméstica y a las técnicas constructivas; sin embargo, la casa circular castreña, la forma de la vivienda y del asentamiento, continúan siendo utilizados a pesar de que la cultura que los dotó de sentido esté cam- biando paulatinamente? ${ }^{9}$ Así se explicaría la coexistencia, en este momento, del tipo de vivienda tradicional con otras casas que recogen las nuevas aportaciones; el reemplazo de las formas arquitectónicas antiguas se debería en muchos casos al valor prestigioso y a la calidad constructiva de la nueva arquitectura, más que a una inutilidad o falta total de adecuación a un modo de vida que se continuaba practicando.

Por otro lado, esta coexistencia habitacional pone de manifiesto a su vez una incipiente ruptura con el modelo tradicional. Las comunidades campesinas entran a formar parte de un nuevo marco económico, caracterizado por la extensión de la actividad agrícola de base cerealística, un incremento de la actividad artesanal y la articulación de redes comerciales que se benefician de las nuevas vías de comunicación construidas por Roma en el territorio galaico. Pero también se integran en un nuevo contexto institucional, religioso e ideológico; a este respecto, la romanización supone una transformación o remodelación profunda en la cosmovisión y en la estructura familiar de la sociedad castreña.

\footnotetext{
9 En Britania el paso de la vivienda circular a la rectangular enmascara una continuidad en la organización básica del espacio; las nuevas casas rectangulares vinieron a ser la expresión, mediante una forma diferente, del modelo conceptual/espacial precedente (HINGLEY, 1990).
} 
La romanización acentuó el poder de la línea masculina en el seno de las unidades familiares (de acuerdo con el modelo patrilineal y patrilocal latino), siendo la que detenta ahora la propiedad de los medios de producción, dentro de un proceso de privatización de la tierra. Se establece así un nuevo modelo que prefigura la familia campesina altomedieval, sancionado ideológicamente por la doctrina cristiana desde el s. IV d.C.

De este modo, creemos que los cambios en la organización interna del espacio doméstico obedecen a una transformación en la estructura económica y familiar; la ruptura de un espacio doméstico único, colectivo, quizás ponga de manifiesto una concepción diferente del individuo. La compartimentación del espacio, organizado en diferentes estancias, reflejaría una mayor especialización económica (diferentes áreas de actividad) y una segmentación en el interior de la unidad familiar (espacios con un alto grado de privacidad). La casa, más integrada dentro de la organización urbanística del poblado, se define todavía más como núcleo identitario, con un control y una restricción más acusada del acceso a la misma.

Se trata de cambios sustanciales, producto de un proceso de aculturación que minó la legitimidad y estabilidad de la inamovible, hasta ese momento, tradición arquitectónica prerromana.

\section{Bibliografía}

ACuña CASTROviejo F., 1996, Urbanismo castreño en el Noroeste peninsular, en C. FERNÁNDEZ OCHOA (coord.), Los Finisterres Atlánticos en la Antigüedad. Época prerromana y romana, Madrid, pp. 45-47.

Allison P. M. (ed.), 1999, The Archaeology of Household Activities, London.

Almagro-Gorbea M., 1994, Urbanismo de la Hispania céltica. Castros y oppida del Centro y Occidente de la Península Ibérica. Castros y Oppida en Extremadura, en M. Almagro y A. M.a Martín (eds.), Castros y Oppida en Extremadura. Complutum, pp. 13-75.

Arias Vilas F., 1985, Castro de Viladonga. Campaña de 1983. Arqueoloxía/Memorias, n.o 2, Santiago.

ARIAS Vilas F., DurÁn Fuentes M. ${ }^{a}$ C., 1996, Museo do Castro de Viladonga (Castro de Rei, Lugo), Santiago.

ARIAS VILAS F., 2000, Os últimos traballos arqueolóxicos no castro de Viladonga: 1988-1998, Brigantium, n. ${ }^{\circ} 12$, pp. 187-198.

AyÁn Vila X. M., 2001, Arqueotectura 2: La vivienda castreña. Propuesta de reconstrucción en el castro de Elviña ( $A$ Coruña), TAPA (Traballos en Arqueoloxía da Paisaxe), n. ${ }^{\circ}$ 5, Santiago de Compostela.

AYÁn VILA X. M., 2002, O estudo da arquitectura doméstica da Idade do Ferro do NW: achega historiográfica, Gallaecia, n. ${ }^{\circ} 21$ : 137-57.

Ayán Vila X. M., Blanco Rotea R., Mañana BorrazÁs P., 2003, Archaeotecture: seeking a new archaeological vision of Architecture, en X. M. Ayán Vila, R. Blanco Rotea, P. Mañana Borrazás (eds.), Archaeotecture: Archaeology of Architecture, Oxford, pp. 1-15.

BAKER G.H., 1998, Análisis de la forma: urbanismo y arquitectura, México. Blanton R. E., 1994, Houses and households. A comparative study, New York.
Carballo ArCeo X., 1996, Os castros galegos: espacio e arquitectura, Gallaecia, n. ${ }^{\circ}$ 14/15, pp. 309-357.

Chamoso Lamas M., 1977, Las excavaciones del Castro de Viladonga y la problemática que plantean sus resultados, en Actas del Coloquio sobre el Bimilenario de Lugo, Lugo, pp. 41-46.

Chapman J., 1990, Social Inequality on Bulgarian tells an the Varna Problem, en R. SAMSON (ed.), The social Archaeology of houses, Edinburgh, pp. 49-92.

ChING F., 1995, Arquitectura: forma, espacio y orden, México.

Clarke D. L., 1972, A provisional model of an Iron Age Society and its settlements system, en D. L. Clarke (ed.), Models in Archaeology, London, pp. 801-869.

Criado BoAdo F., 1993, Visibilidad e interpretación del registro arqueológico, Trabajos de Prehistoria, n. ${ }^{\circ}$ 50, pp. 39-56.

Criado Boado F., 1999, Del Terreno al Espacio: Planteamientos y Perspectivas para la Arqueología del Paisaje, CAPA (Criterios y Convenciones en Arqueología del Paisaje), n. ${ }^{\circ}$ 6, Santiago.

de la Peña Santos A., 1988, Algunos aspectos urbanísticos del poblado galaico-romano de Santa Tegra (A Guarda-Pontevedra), Pontevedra. Revista de Estudios Provinciales, n. ${ }^{\circ}$ 4, pp. 27-36.

FERNÁNDEZ-POSSE M. ${ }^{a}$ D., 1998, La investigación protohistórica en la Meseta y Galicia, Madrid.

Fernández-Posse M. a D., SÁnChez Palencia F. J., 1998, Las comunidades campesinas en la cultura castreña. Trabajos de Prehistoria, 55 (2): 127-150.

FOSTER S., 1989, Analysis of spatial patterns in buildings (access analysis) as an insight into social structure: examples from the Scottish Atlantic Iron Age, Antiquity, n. ${ }^{\circ}$ 63, pp. 40-50.

Hillier B., HANSON J., 1984, The social logic of space, Cambridge.

Hingley R., 1990, Domestic Organization and Gender Relations in Iron Age and Roman-British Households, en R. SAMSON (ed.), The Archaeology of houses: 125-47. Cambridge: Cambridge University Press.

HODDER I., 1994, Architecture and Meaning: The Example of Neolithic Houses and Tumbs, en M. Parker Pearson y C. Richard (eds.), Architecture and Order. Approaches to Social Space, London, pp. 73-86.

JOHNSON M., 1993, Housing Culture. Traditional architecture in an English landscape, London.

KENT S. (ed.), 1990, Domestic Architecture and the Use of Space. New Directions in Archaeology, Cambridge.

Llana Rodríguez C., Varela Arias E., 1999, Una aproximación a las actividades realizadas en la zona oriental del Barrio Norte del Castro de Viladonga (Castro de Rei, Lugo), Croa, n. ${ }^{\circ}$ 9, pp. 14-29.

Llana Rodríguez C., Varela Arias E., 2000, Acerca de la funcionalidad de la zona occidental del barrio norte del Castro de Viladonga, Croa, n. ${ }^{\circ} 10$, pp. 21-32.

Llana Rodríguez C., Varela Arias E., 2001, Las primeras excavaciones en el castro de Viladonga, treinta años después. Acerca de la funcionalidad de la zona occidental del barrio norte del Castro de Viladonga, Croa, n. ${ }^{\circ} 11$, pp. 11-24.

Mañana BorrazÁs P., Blanco Rotea R., Ayán Vila X.M., 2002, $A r-$ queotectura 1: Bases teórico-Metodológicas para una Arqueología de la Arquitectura, TAPA (Traballos en Arqueoloxía da Paisaxe), n.o 15, Santiago de Compostela.

RAPOPORT A., 1972, Vivienda y cultura, Barcelona.

SAMSON R. (ed.), 1990, The social Archaeology of houses, Edinburgh.

SÁnCHEZ J., 1998, La Arqueología de la Arquitectura. Aplicación de nuevos modelos de análisis a estructuras de la Alta Andalucía en época ibérica, Trabajos de Prehistoria, n. 55 (2), pp. 89-109.

Ucko P. J., Tringham R, Dimbley G. W. (eds), 1972, Man, settlement and urbanism, London.

Wilk R., RahtJe W., 1982, Household Archaeology, American Behavioral Scientist, n. ${ }^{\circ} 25$, pp. 617-639. 